\title{
Análise do gerenciamento de resíduos do centro cirúrgico de um Hospital Universitário do Estado do Rio de Janeiro
}

\author{
Analysis of waste management in the operating room of a university \\ hospital in Rio de Janeiro
}

Maithê Lemos' ${ }^{1}$ Joanir Passos², Dolly Arias Torres ${ }^{3}$

\begin{abstract}
Resumo
Os resíduos hospitalares, se descartados sem o devido tratamento ou ainda sem a observância das leis existentes, podem constituir perigo à população, aos profissionais de saúde e aqueles que participam do processo de manejo dos mesmos. Objetivou-se descrever e mapear o processo de gerenciamento de resíduos do Centro Cirúrgico, de um Hospital Público da cidade do Rio de Janeiro, confrontando com as exigências legais. $O$ estudo em questão foi realizado através de observação nâo participante. $O$ instrumento de coleta de dados utilizados na observaçâo não participante foi extraído de uma tese, com a devida autorizaçāo da autora. Verificou-se que nem todos os quesitos observados pela pesquisadora estavam em consonância com a legislaçâo vigente e ainda que as condutas adotadas levam ao fluxo de resíduos com falhas consideráveis a serem resolvidas, como o cruzamento de materiais limpos e resíduos contaminados.
\end{abstract}

Descritores: Enfermagem, gerenciamento de resíduos, resíduos de serviços de saúde, resíduos sólidos.

\begin{abstract}
Clinical waste, if disposed without proper treatment or even without compliance of existing laws, may constitute a danger to the public, health professionals and those involved in the process of handling them. This study aimed to describe and map the process of waste management in the Surgical Center, of a public hospital in the city of Rio de Janeiro, confronting them with legal requirements. This
\end{abstract}

study was conducted through non-participant observation. The data collection instrument used in the non-participant observation was taken from a thesis, with author's permission. It was verified that not all the points observed by the researcher were in line with current legislation, and that the approaches adopted takes to the waste stream with considerable failure to be resolved, such as the intersection of clean and contaminated waste materials.

Keywords: Nursing, waste management, waste health services, solid waste.

\section{Resumen}

La eliminación de residuos sanitarios sin tratamiento adecuado y sin cumplir con las leyes existentes, puede constituir un peligro para la población en general, los profesionales de la salud y para quienes participan en el manejo de los mismos. Metodología, Estudio observacional con el objetivo de describir y mapear el proceso de gestión de residuos del Centro Quirúrgico de un hospital público de Río de Janeiro y el cumplimiento de los requisitos legales. Se utilizó la observación no participante y el instrumento de recolección de datos fue extraído de una tesis, con el permiso del autor. Resultados, se encontró que no todos los pasos observados estaban en consonancia con la legislación vigente y que las conductas adoptadas denotaban la presencia de defectos considerables, tales como la combinación de residuos limpios con los contaminados.

Palabras clave: Enfermería, gestión de residuos, residuos en servicios de salud, residuos sólidos.

1 Enfermeira do Trabalho, Mestre em Enfermagem, Doutoranda em Enfermagem e Biociencias da Universidade Federal do Estado do Rio de Janeiro, Integrante do Laboratório de Pesquisa: Enfermagem, Tecnologias, Saúde e Trabalho, e-mail: mait_lemos@yahoo.com.br

2 Doutora em Enfermagem, Coordenadora do Programa de Pós-graduação em Enfermagem - PPGENF, Professora Associada Escola de Enfermagem Alfredo Pinto, Universidade Federal do Estado do Rio de Janeiro (UNIRIO), Líder do Integrante do Laboratório de Pesquisa: Enfermagem, Tecnologias, Saúde e Trabalho, e-mail: joppassos(ahotmail.com

3 Enfermeira, Mestre em Educação e Desenvolvimento Comunitário, Doutora em Ciências da Saúde, Professora Titular da Universidade Surcolombiana, Integrante do Grupo de Pesquisa Cuidar, e-mail: dolaria@usco.edu.co

Recibido: 15/12/2014 - Revisado: 23/12/2014 - Aceptado: 24/02/2015 
R.F.S Revista Facultad de Salud

Julio - Diciembre de 2014;6(2): 7-11
Análise do gerenciamento de resíduos do centro cirírgico de um Hospital Universitário do Estado do Rio de Janeiro/Maithê Lemos et al.

\section{Introdução}

No Brasil, a maior parte dos resíduos é descartada no meio ambiente sem qualquer tipo de tratamento, provocando o adoecimento da população e a desestruturação do ecossistema. Com os Resíduos de Serviços de Saúde (RSS) não é diferente, além de provocarem acidentes no trabalho pelo manejo inadequado, o resíduo sem tratamento pode ser fonte de contaminação provocando um alerta do ponto de vista epidemiológico, sanitário, ocupacional, ambiental e social.

De acordo com a Resolução No 283 do Conselho Nacional do Meio Ambiente (CONAMA) os RSS são: a) aqueles provenientes de qualquer unidade que execute atividades de natureza médicoassistencial humana ou animal; $(\ldots)^{[1: 1]}$

O Programa de Gerenciamento de Resíduos de Serviços de Saúde (PGRSS) requer um estudo detalhado de cada unidade hospitalar, e, o retrato desta situaçẫo pode contribuir para o planejamento e elaboração das medidas a serem tomadas na reestruturação deste sistema além de promover a higiene, segurança e a saúde no trabalho, evitar acidentes com os trabalhadores e minimizar os riscos ocupacionais.

Neste sentido o objetivo deste estudo foi descrever e mapear o processo de gerenciamento de resíduos do Centro Cirúrgico (CC) de um hospital público, confrontando com as exigências legais.

\section{Material e Métodos}

Trata-se de um recorte da dissertação de mestrado apresentada na Universidade Federal do Estado do Rio de Janeiro, intitulada "Gerenciamento de Resíduos de um Hospital Público do Rio de Janeiro: um estudo sobre o saber/fazer da enfermagem no Centro Cirúrgico e Central de Materiais." Para este artigo optou-se por um estudo descritivo observacional, favorecendo o levantamento e diagnóstico da situaçẫo do gerenciamento de resíduos desde seu descarte inicial, na fonte geradora até a coleta fora do hospital. ${ }^{[2]}$

A pesquisa foi realizada no CC de um hospital público de médio porte, situado na cidade do Rio de Janeiro. A coleta de dados foi realizada através da observação não participante, seguindo o instrumento validado utilizado por Magda Fabbri Isaac Silva em sua tese de doutorado, previamente solicitado o uso e aceito por e-mail. No momento da observação não participante foi realizado um registro fotográfico do ambiente, previamente autorizado pela Instituição, objetivando identificar os descartes dos resíduos na fonte geradora.

É importante ressaltar que todos os cuidados relativos aos aspectos éticos envolvidos na pesquisa foram devidamente observados, aprovado pelo Comitê de Ética em Pesquisa da própria Instituição (Protocolo no 86437), em atendimento ao disposto na antiga Resolução N 196/96 do Conselho Nacional de Saúde (atual Resolução no 466/2012).

A coleta dos dados ocorreu nos meses de outubro e novembro de 2012, as observaçôes duraram em média duas horas cada e foram realizadas até que o cenário passou a se repetir. A análise dos dados foi realizada por meio da ferramenta de fluxograma analitico, um instrumento de análise que se propõem a interrogar o processo de trabalho, revelando a maneira de organizá-1o. ${ }^{[3]}$

\section{Resultados}

O CC dispồe de sete salas de cirurgias, um lavatório que antecede as salas para higienização das mãos, um expurgo, dois arsenais, vestiários (feminino e masculino) com respectivos banheiros e copa.

Os resíduos infectantes gerados nas salas do CC eram provenientes de procedimentos realizados durante as cirurgias. Foi observado que, no momento das cirurgias, dois hampers sem identificação de conteúdo eram utilizados para descarte de resíduos comuns e roupas de cama/tecidos. E em outros momentos, foi possível perceber que em um mesmo recipiente havia dois sacos para acondicionamento de resíduos diferentes sobrepostos, conforme figura 1.

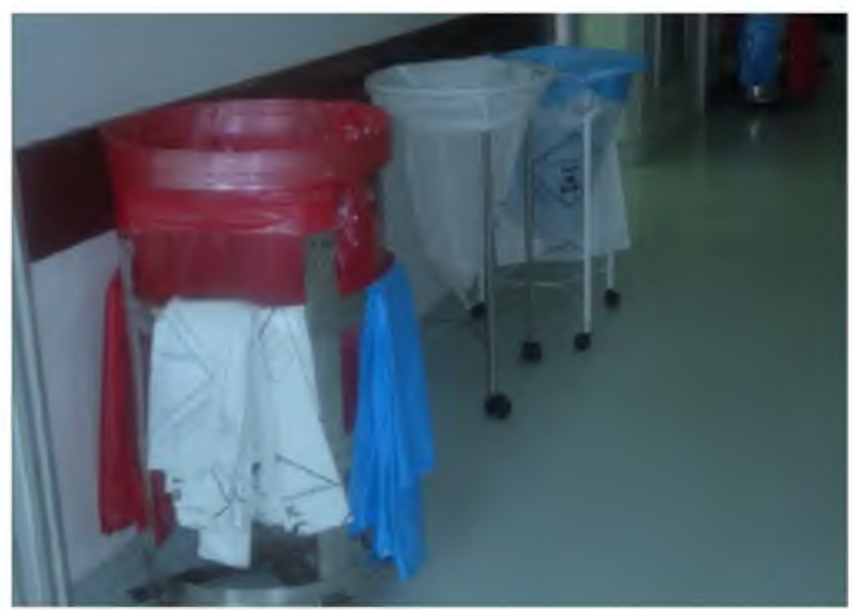

Figura 1. Hampers no corredor do Centro Cirúrgico, com sacos de cores diferentes e mistura de sacos no mesmo hamper, dificultando a identificação do recipiente.

Houveram desvios de comportamento no ato do descarte com direcionamento inapropriado de diversos tipos de resíduo a um mesmo saco. Assim, os resíduos comuns e os infectantes se misturavam com as roupas, de acordo com a figura 2 . Devido a esta mistura indiscriminada de resíduos, após as cirurgias, as funcionárias da limpeza realizavam a higienização das salas e separavam os resíduos, antes misturados, direcionando-os aos recipientes adequados.

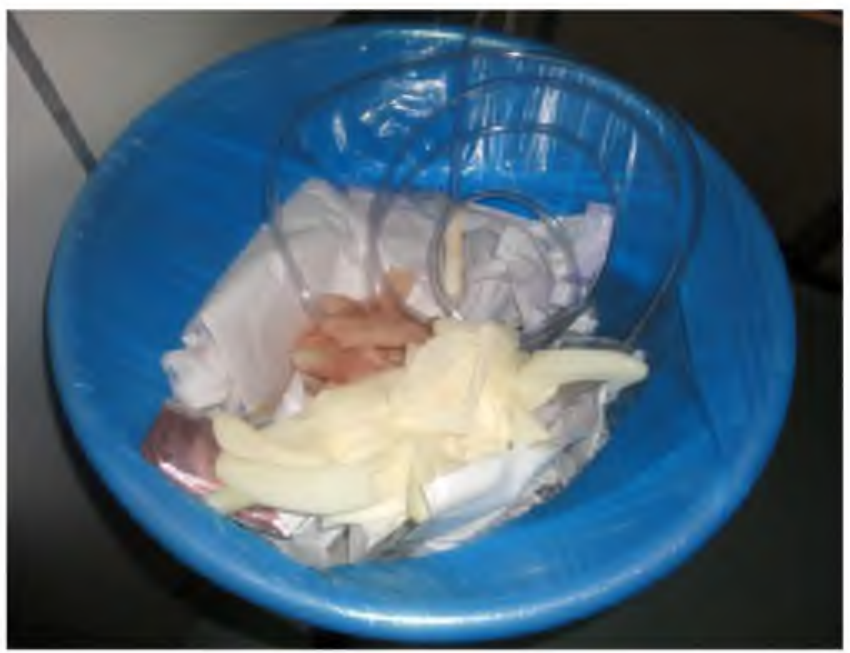

Figura 2. Hamper com saco azul, sem identificação. Em seu interior consta resíduos comuns, infectantes e tecidos misturados. 
No decorrer de procedimentos cirúrgicos, os resíduos pérfurocortantes foram, majoritariamente, descartados pelo anestesista na caixa destinada a este fim. No entanto, quando isto não ocorria, o circulante de sala encarregava-se de retirar os pérfurocortantes separados, pelo anestesista, na bandeja de procedimentos.

Todas as salas do CC possuíam uma caixa para péfurocortantes afixadas na parede, porém longe da mesa cirúrgica. Em algumas observaçồes, notou-se que os resíduos excediam o limite de cinco centímetros abaixo do bocal da caixa.

Apôs as cirurgias era possível perceber resíduos infectantes, resíduos comuns e roupas de cama espalhadas pelo chẫo da sala, caracterizando a desorganização do processo de trabalho, conforme figura 3 .

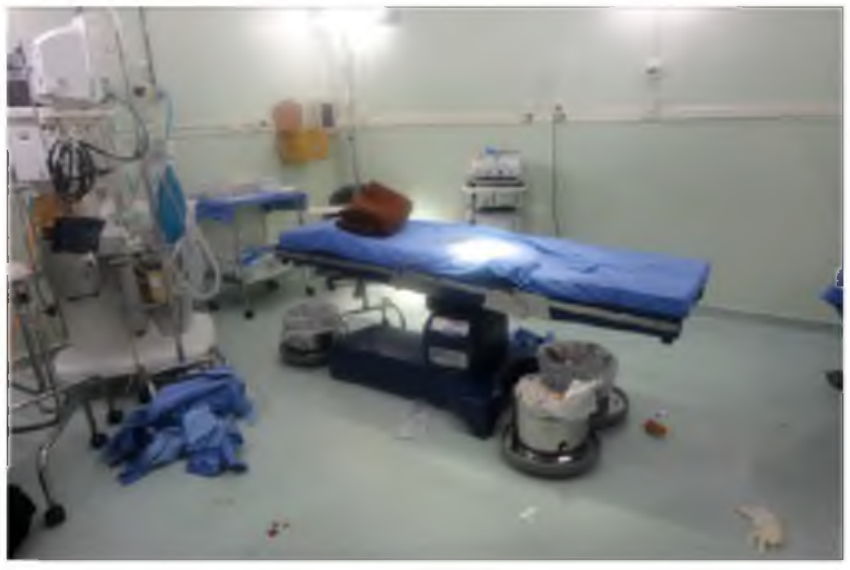

Figura 3. Sala cirúrgica após a realização do procedimento, evidenciando resíduos pelo chão e desorganização do processo de trabalho.

Em relação aos resíduos químicos observados no $\mathrm{CC}$, os mesmos também eram descartados na caixa de pérfurocortantes. Os restos de formaldeído utilizados para manutenção de peças anatômicas em eventual retirada, álcool e PVP-I eram descartados juntamente com os fluidos corporais em balde, levados até o expurgo pela enfermagem, com as mãos enluvadas, para descarte no tanque e lançamento direto no esgoto, conforme figura 4.

O expurgo possui dois tanques, utilizados para descarte de fluidos, excretas de pacientes, lavagem de panos, de mãos e de recipientes. Possui ainda, uma caixa para resíduos pérfurocortantes, além de materiais para limpeza, acondicionados em galões identificados, no chão.

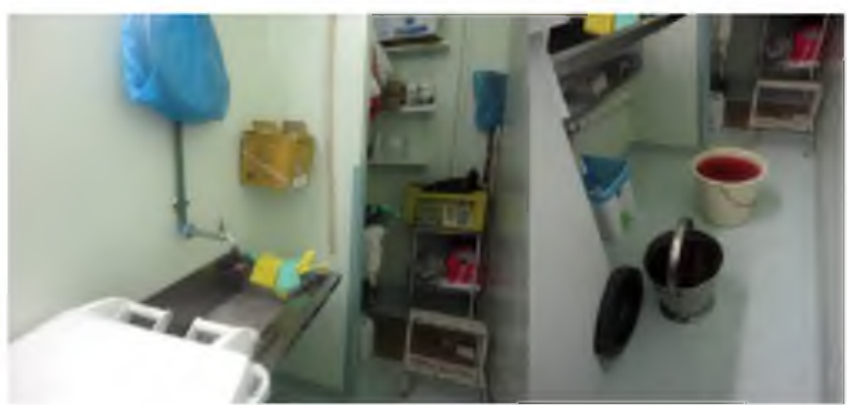

Figura 4. Tanque do expurgo com o sangue e fluidos corporais desprezados diretamente na rede de esgoto.
No expurgo há um carro com tampa e identificação para resíduos infectantes, que armazena temporariamente e transporta até a saída, todos os resíduos coletados (comuns e infectantes), conforme figura 5. Nas observações foi possível perceber que a grande quantidade de resíduos gerada não é comportada pelo pequeno espaço do expurgo e pelo único carro de transporte, sendo necessária a condução dos sacos contendo resíduos infectantes com o uso das mãos, ocorrendo o contato desses resíduos com outras partes do corpo.

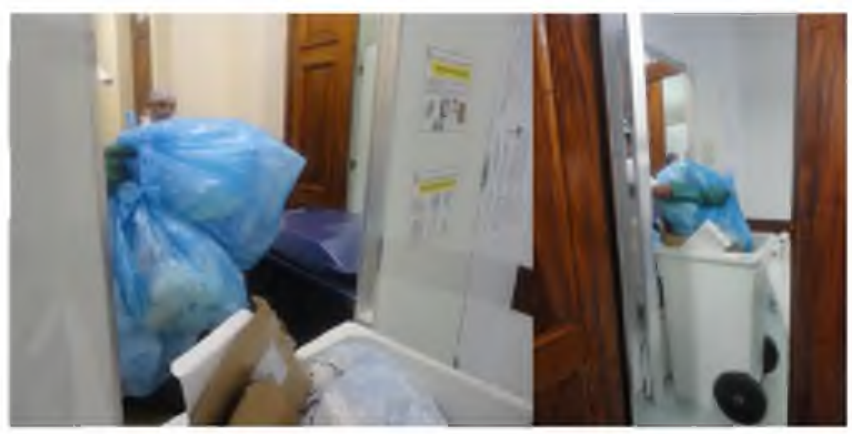

Figura 5. Diversos tipos de resíduos armazenados e transportados pelo mesmo carro de transporte, identificado para transporte de resíduos infectantes.

É importante ressaltar que existe somente uma saída no $\mathrm{CC}$, portanto, o mesmo local onde saíam materiais sujos entravam materiais esterilizados, sendo o mesmo local por onde entravam e saíam pacientes e os resíduos do setor. O fluxograma dos resíduos gerados no CC, está sintetizado na figura 6 .

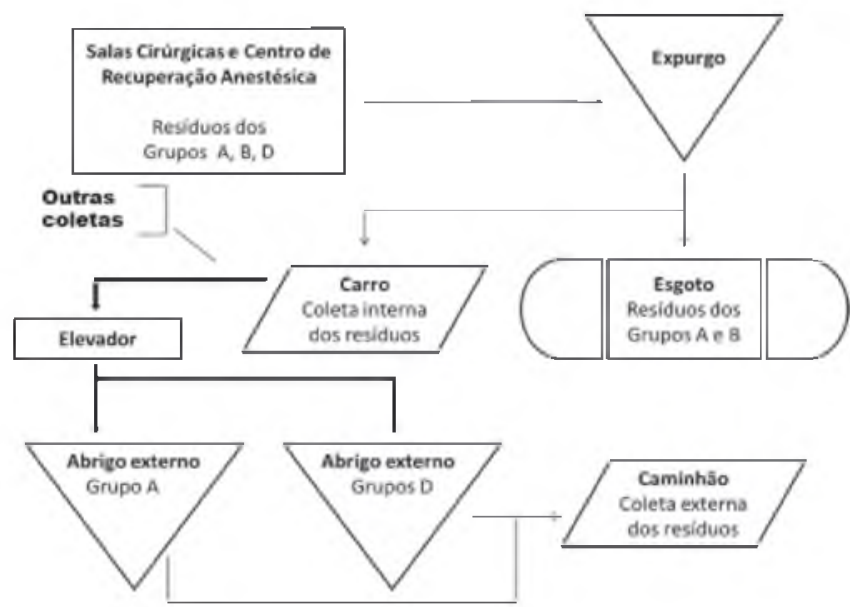

Figura 6. Fluxograma de Resíduos do Centro Cirúrgico

\section{Discussão}

O desvio de conduta dos profissionais de saúde e da limpeza apareceu maciçamente nas observaçôes realizadas. A mistura de residuos em um mesmo recipiente é inconcebível, já que posteriormente sâo acondicionados e tratados como resíduos comuns, pois mesmo que haja posterior separação desses resíduos, não se elimina o risco da contaminaçẫo de todo o material, ou mesmo parte dele, uma vez que já tenha ocorrido contato com o resíduo infectante. ${ }^{[4,5]}$ 
R.F.S Revista Facultad de Salud

Julio - Diciembre de 2014;6(2): 7-11
Análise do gerenciamento de resíduos do centro cirírgico de um Hospital Universitário do Estado do Rio de Janeiro/Maithê Lemos et al.
Cabe salientar que tornar todos os resíduos infectantes, através da mistura, pode promover o aumento de custos à Instituição, visto que o tratamento dado aos resíduos infectantes é mais dispendioso do que o dispensado aos resíduos comuns.

Portanto, é necessàrio questionar se os profissionais desconhecem como realizar a segregação dos resíduos ou se existe omissẫo e talvez certo descaso com o gerenciamento, já que este tipo de atitude é inaceitàvel em qualquer serviço de saúde, quer seja dotado de PGRSS ou nầo. É importante atentar para o fato de que antes do descarte dos resíduos, faz-se mentalmente a separação dos resíduos em classes para posterior segregação em recipientes adequados. A segregação significa separação dos diferentes grupos residuos na fonte geradora, pelo próprio funcionàrio que o gerou. ${ }^{[6]}$

Outras tensões relativas ao descarte são relacionadas aos recipientes existentes para o acondicionamento. Os recipientes que acondicionam os resíduos pérfurocortantes devem ser de material rígido, afixada em suporte próprio de forma que possibilite a visualização do seu interior para o controle do limite da capacidade. Para que possa ser segura e adequadamente fechada, preconiza-se que os resíduos estejam a cinco centímetros do orifício ou preenchidos até $2 / 3$ da capacidade da caixa, de acordo com a NR32 e RDC N $N^{\circ} 306$ da Agência Nacional de Vigilância Sanitària (ANVISA). ${ }^{[7,8]}$

Este estudo aponta, ainda, outra situaçầo alarmante referente ao descarte dos resíduos líquidos. Todos os efluentes líquidos do hospital em estudo são eliminados na rede pública de esgoto sem tratamento prévio. As Resoluções $\mathrm{N}^{\circ} 283,358$ e 05 do CONAMA e a RDC N $\mathrm{N}^{\circ} 306$ da ANVISA consideram o sistema de tratamento de resíduos como um conjunto de unidades, processos e procedimentos que alterem as características visando à minimização do risco à saúde pública, a preservaçâo da qualidade do meio ambiente, a segurança e a saúde do trabalhador. A RDC N 33 da ANVISA, completa que estes métodos devem levar à redução ou eliminação do risco de causar doenças a população. ${ }^{[1,9,10]} \wedge$ Resolução $\mathrm{N}^{\circ} 283$ do CONAMA preconiza que os efluentes líquidos provenientes dos estabelecimentos prestadores de serviços de saúde, devem atender às diretrizes estabelecidas pelos órgãos ambientais. ${ }^{[1]}$

Em relação à coleta e transporte de resíduos, pode ser realizado manualmente desde que o recipiente de segregação nẫo entre em contato com outra parte do corpo, é vedado o arrasto, de acordo com a NR32.7 A coleta e o transporte interno deve ser realizado em sentido único, com roteiro definido, em horários estipulados, de forma a não coincidir com a distribuição de roupas, alimentos e medicamentos, período de visita ou de maior fluxo de pessoas, porém em diversos momentos isto não ocorreu jà que o $\mathrm{CC}$ é movimentado na maior parte do tempo e existe somente uma porta de entra/saída. $[8,10]$

De acordo com a NR32, sempre que o transporte do recipiente de segregação dos RSS comprometerem a segurança e a saúde do trabalhador, devem ser utilizados meios técnicos apropriados, de modo a preservar sua saúde e integridade física. 70 carro de transporte de resíduos utilizado está de acordo com as recomendações da RDC N 306 da ANVISA no que diz respeito à resistência e rigidez do material, lavável, impermeável, provido de tampa articulada, canto e bordas arredondadas, porém nẫo corresponde à norma quando a mesma exige que o recipiente seja identificado com o símbolo correspondente ao risco do resíduo nele contido.

Assim como a classificação dos resíduos, a coleta, o armazenamento e o tratamento é possível afirmar que o gerenciamento de RSS como um todo é extremamente difícil em virtude de vários aspectos. Este processo deve considerar os fatores como: controle das infecções, conservaçâo do meio ambiente e a saúde ocupacional sem deixar de tratar com relevância a manipulaçâo de produtos perigosos, como medicamentos e reagentes químicos.

Neste sentido, deveriam haver parcerias, onde o Estado disponibilizaria um sistema adequado com infraestrutura para a eliminação dos resíduos e para tratamento da água de abastecimento e dos efluentes, como também estar integrado com os órgãos de fiscalização ambiental no sentido de controlar periodicamente os serviços de saúde. ${ }^{[11]}$

O gerenciamento de resíduos do $\mathrm{CC}$ do hospital estudado encontra-se com falhas em várias etapas do processo, porém existem soluções passíveis de resolver o problema instalado. Pensar nas questôes relacionadas aos resíduos ultrapassa as portas dos serviços de saúde é necessário conceber que nossas atitudes no momento inicial da geração dos resíduos, se refletem no momento final de descarte no meio ambiente, perpassando por todo o processo e influenciando no mesmo.

Deste modo, como é possível afirmar que ao final do processo os resíduos são comuns, já que existe maciçamente o desvio de conduta no descarte dos mesmos? Isto é, se ocorrem a mistura de resíduos infectantes e comuns, na fonte geradora e descarte inicial, não é possível afirmar que os resíduos encaminhados à coleta seletiva pelo hospital são verdadeiramente isentos de perigo, no que se refere ao risco biológico e de contaminaçâo de quem o manuseia.

Para o bom funcionamento do PGRSS é necessário considerar açỗes que tenderẫo a desenvolver uma relação favorável e de equilíbrio entre o homem, meio ambiente, sociedade e também as instituições hospitalares, uma vez que trarâo impactos benéficos nos aspectos biossanitários, ambientais, sociais, tecnológicos, culturais, econômicos e legais.

Portanto, hà uma imperiosa necessidade do cumprimento criterioso às normas legais estabelecidas para o gerenciamento de resíduos, dando ênfase aos de saúde pública, saúde do trabalhador, aspectos ambientais e epidemiológicos, com o propósito de que se unidos, alcançarão resultados significativos para saúde humana e ambiental.

\section{Referências}

1. Conselho Nacional do Meio Ambiente. Resolução n.o 283, de 1 de outubro de 2001. Dispỗem sobre o tratamento e destinação final dos resíduos de serviços de saúde. Diário Oficial da República Federativa do Brasil, 01 out 2001 ; Seção 1.

2. Polit DF, Beck CT. Fundamentos da Pesquisa em Enfermagem. 8.ed. Sẵo Paulo: Art Med; 2011.

3. Barbosa TAV, Fracolli LA. A utilização do "fluxograma analisador" para a organização da assistência à saúde no Programa Saúde da Família. Cadernos de Saúde Pública. 2005;21:1036-1044.

4. Dias SM, Figueiredo LCA. Educação Ambiental como estratégia para a redução de resíduos de serviços de saúde em hospital de Feira de Santana. In: Congresso Brasileiro de Engenharia Sanitária e Ambiental; 1999; Rio de Janeiro, BR. Rio de Janeiro: Associação Brasileira de Engenharia Sanitária e Ambiental; 1999. p. 2615 2620. 
5. Coelho NMGP Gerenciamento de resíduos de serviços de saúde: manejo dos resíduos potencialmente infectantes e pérfurocortantes em unidades de internação da criança, adulto e pronto-socorro de hospitais públicos no distrito federal [Dissertação de Mestrado]. Brasília: Universidade de Brasília; 2007.

6. Salles CLS. Acidentes de trabalho ocorridos com os trabalhadores da saúde nos diferentes processos de um plano de gerenciamento de resíduos de serviços de saúde [Dissertação de Mestrado]. Guarulhos: Universidade Guarulhos, 2008.

7. Ministério do Trabalho e Emprego. Norma Regulamentadora n.o 32, de 16 de novembro de 2005. Dispōem sobre a Segurança e Saúde no trabalho em serviços de saúde. Diário Oficial da União, 16 nov 2005.

8. Agẽncia Nacional de Vigilância Sanitária. Resolução RDC 306, de 7 de dezembro de 2004. Dispõe sobre o regulamento técnico para o gerenciamento de resíduos de serviços de saúde. Diário Oficial da União, 7 dez 2004
9. Conselho Nacional do Meio Ambiente. Resoluçẫo n. 358, de 4 de maio de 2005. Dispôe sobre o tratamento e a disposição final dos resíduos dos serviços de saúde e dá outras providências. Diário Oficial da República Federativa do Brasil, 4 de mai 2005; Seção 1:63.

10. Agência Nacional de Vigilância Sanitária. Resolução RDC 33, de 25 de fevereiro de 2003. Dispōe sobre o Regulamento Técnico para o gerenciamento de resíduos de serviços de saúde. Diário Oficial da União, 5 mar 2003.

11. Machline C, Gonçalves R, Ribeiro Filho V O Gerenciamento dos Resíduos dos Serviços de Saúde de Uma Amostra de Hospitais Nacionais. In: Anais do Simpósio de Administração da Produção, Logística e Operaçōes Internacionais; 2005; São Paulo, BR. São Paulo: Fundação Getúlio Vargas; 2005. p. 8. 